\title{
MiR-363 inhibits cisplatin chemoresistance of epithelial ovarian cancer by regulating snail-induced epithelial-mesenchymal transition
}

\author{
Lanqin Cao ${ }^{1}$, Qian Wan ${ }^{1}$, Fengjie $\mathrm{Li}^{1}$ E Can-e Tang ${ }^{2, *}$ \\ ${ }^{1}$ Department of Obstetrics, Xiangya Hospital, Central South University, Changsha, Hunan $410008,{ }^{2}$ The Institute of Medical Science \\ Research, Xiangya Hospital, Central South University, Changsha, Hunan 410008, P. R., China
}

\begin{abstract}
Chemoresistance is a major barrier to successful cisplatinbased chemotherapy for epithelial ovarian cancer (EOC), and emerging evidences suggest that microRNAs (miRNAs) are involved in the resistance. In this study, it was indicated that miR-363 downregulation was significantly correlated with EOC carcinogenesis and cisplatin resistance. Moreover, miR-363 overexpression could resensitise cisplatin-resistant EOC cells to cisplatin treatment both in vitro and in vivo. In addition, data revealed that EMT inducer Snail was significantly upregulated in cisplatin-resistant EOC cell lines and EOC patients and was a functional target of miR-363 in EOC cells. Furthermore, snail overexpression could significantly attenuate miR-363-suppressed cisplatin resistance of EOC cells, suggesting that miR-363-regulated cisplatin resistance is mediated by snail-induced EMT in EOC cells. Taken together, findings suggest that miR-363 may be a biomarker for predicting responsiveness to cisplatin-based chemotherapy and a potential therapeutic target in EOC. [BMB Reports 2018; 51(9): 456-461]
\end{abstract}

\section{INTRODUCTION}

Ovarian cancer (OC) is the second most common cancer and a leading cause of death from gynaecologic malignancies in women worldwide (1). Epithelial ovarian cancer (EOC) is the most common type and deadly form of high-grade serous OC (2). The standard treatment for EOC is debulking surgery, followed by platinum-based chemotherapy (3). Cisplatin, a platinum compound, is one of the most common first-line antitumour agents, which bind to and cross-link DNA in

${ }^{*}$ Corresponding author. Tel: +86-0731-84327628; Fax: +86-73184327332; E-mail: csutangcane@163.com

https://doi.org/10.5483/BMBRep.2018.51.9.104

Received 8 May 2018, Revised 29 May 2018, Accepted 20 July 2018

Keywords: Chemoresistance, Cisplatin, Epithelial-mesenchymal transition, Epithelial ovarian cancer, MiR-363, Snail cancer cells $(4,5)$. However, due to cisplatin resistance, the overall 5-year survival rate of EOC patients is about $40 \%$ (6). Accumulating evidence demonstrated that processes of the epithelial-mesenchymal transition (EMT), which promote cancer progression and metastasis, played a role in the development of chemoresistance (7). In EOC, Marchini et al. indicated that several genes involved in EMT were associated with overall or progression-free survival, suggesting that EMT was vital to resistance mechanisms (8).

MicroRNAs (miRNAs) are endogenous small non-proteincoding RNA molecules with approximately 22 nucleotides, which usually function as negative regulators of gene expression via binding to targeted mRNA (9). Numerous studies demonstrated that miRNAs have been involved in various biological processes, including cell proliferation, homeostasis, cellular differentiation and tumorigenesis (10-12). It's have been reported that miR-363 acts as a tumor suppressor in thyroid (13), gastric (14), colorectal (15), breast (16), and renal (17) cancers. Besides, miR-363 plays an oncogenic role in prostate cancer (18) and glioma (19). In OC, low miR-363 levels were associated with advanced stage, lymph node metastasis, and poor prognosis, meanwhile, miR-363 could inhibit OC cell growth, migration and invasion (20). Recent evidence indicates that miR-363 play important roles in chemoresistance to multiple anticancer drugs. For example, miR-363 promoted resistance to doxorubicin + cisplatin + 5-FU in gastric cancer via targeting FBW7 (14); In breast cancer, miR-363 reversed the resistance to cisplatin by negative regulating of $\mathrm{Mcl}-1$, which is an anti-apoptotic Bcl-2 family member and often overexpresse in breast tumors (16); miR-363 reduced oxaliplatin resistance by targeting the 3 '-UTR of NR2F1-AS1 and ABCC1 mRNA in hepatocellular carcinoma (21). However, whether miR-363 modulate cisplatin resistance in EOC and the mechanisms underlying the resistance remains to be fully understood.

In this study, qRT-PCR assay was applied to assess miR-363 expression in primary cisplatin-sensitive and cisplatin-resistant EOC patients and tissues. In addition, in vitro and in vivo functional studies of miR-363 were conducted to determine their potential roles in the regulation of cisplatin resistance.

ISSN: 1976-670X (electronic edition)

Copyright (C) 2018 by the The Korean Society for Biochemistry and Molecular Biology

(c) This is an open-access article distributed under the terms of the Creative Commons Attribution Non-Commercial License (http://creativecommons.org/licenses/by-nc/4.0) which permits unrestricted non-commercial use, distribution, and reproduction in any medium, provided the original work is properly cited. 
Furthermore, data indicated that miR-363 might directly target Snail, and this interaction played an important role in the regulation of chemoresistance in EOC.

\section{RESULTS}

\section{Decreased miR-363 is associated with EOC} tumourprogression and cisplatin chemoresistance miR-363 expression levels were evaluated in 107 malignant EOC tissues resected at the time of primary surgery from patients who subsequently received cisplatin-based primary therapy and 29 benign tissue samples. qRT-PCR revealed that miR-363 expression levels were significantly decreased in malignant EOC tissues compared with benign tissues (Fig. 1A). Correlation analysis in 107 patients with malignant EOC further revealed that miR-363 downregulation was significantly correlated with high FIGO stage $(P=0.03)$, metastasis $(P=$ $0.011)$ and chemoresistance $(P=0.006)$ (Supplementary Table 1). $83.3 \%$ of the patients with high miR-363 expression levelsand only $56.9 \%$ of the patients with low miR-363 expression levels had primary chemosensitivity. miR-363 relative expression levels in chemoresistant group were significantly lower than that of chemosensitive group (Fig. 1B). In order to substantiate the involvement of miR-363 in cisplatin response in vitro, miR-363 expression levels and cisplatin sensitivity in chemoresistant OVC cells A2780cp and C13 as well as their chemosensitive counterparts A2780s and OV2008 were evaluated. qRT-PCR data revealed that the levels of miR-363 were significantly decreased in A2780cp and C13 cells compared to that of A2780s and OV2008 cells (Fig. 1C). The half maximal (50\%) inhibitory concentration

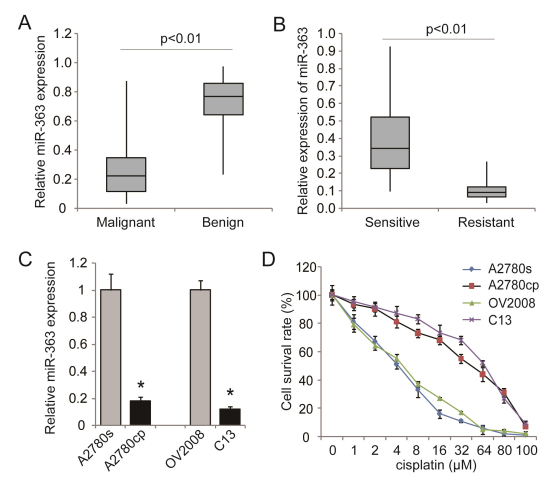

Fig. 1. Decreased miR-363 levels in cisplatin-resistant EOC patients. (A) qRT-PCR analysis of miR-363expression levels in 107 malignant EOC tissues and 29 benign tissues. (B) qRT-PCR analysis of miR-363 expression levels in primary cisplatin-sensitive ( $\mathrm{n}=72)$ and cisplatin-resistant $(\mathrm{n}=35)$ EOC tissues. (C) qRT-PCR analysis of miR-363 expression levels in chemoresistant OVC cells A2780cp and C13 and their chemosensitive counterparts A2780s and OV2008 cells. (D). MTT assay of cisplatin sensitivity in A2780cp, C13, A2780s and OV2008 cells $(* P$ value $<0.05)$.
(IC50) values were significantly higher in A2780cp and C13 cells compared with A2780s and OV2008 cells (Fig. 1D). Taken together, data suggest that decreased miR-363 may be associated with EOC carcinogenesis and cisplatin resistance.

\section{miR-363 sensitizes EOC cells to cisplatin treatment in vitro and in vivo}

To investigate the role of miR-363 in regulating cisplatin sensitivity in EOC cells, miR-363 has been restored in the cisplatin-resistant cells A2780cp and C13 and has been knocked down in the cisplatin-sensitive cells A2780s and OV2008, respectively (Fig. 2A). Enhancing the expression of miR-363 in A2780cp and C13, cell migration and invasion were repressed (Fig. S1). MTT assays with different cisplatin doses showed that cisplatin sensitivities were significantly increased after forced miR-363 overexpression in A2780cp and $\mathrm{C} 13$ cells (Fig. 2B). Conversely, the cisplatin sensitivities of A2780s and OV2008 cells were decreased after miR-363 silencing (Fig. 2C). To further evaluate the role of miR-363 in regulating cisplatin sensitivity in vivo, the cisplatin-resistant cells A2780cp were injected subcutaneously, overexpressing

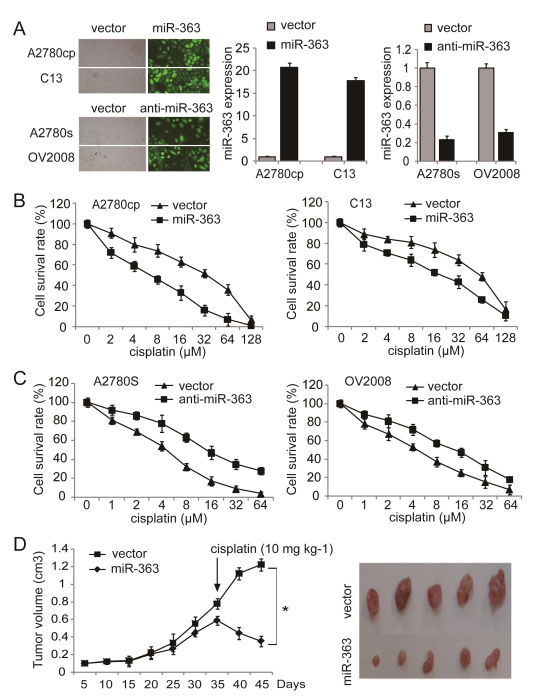

Fig. 2. Restoration of miR-363 sensitizes EOC cells to cisplatin in vitro and in vivo. (A) The chemoresistant A2780cp and $\mathrm{C} 13$ and chemosensitive A2780s and OV2008 cells were infected with Lv-miR-363 and Lv-NC labelled with GFP reporter, respectively. qRT-PCR was performed to determine miR-363 expression levels. $(B, C)$ The impacts of miR-363 on cisplatin sensitivity at different doses $(0,2,4,6,8$ and $10 \mathrm{ng} / \mathrm{ml})$ were determined by MTT assay. (D) BALB/C nude mice were subcutaneously inoculated with Lv-miR-363-infected A2780cp cells $(\mathrm{n}=5)$ and Lv-miR-NCinfected A2780cp cells $(\mathrm{n}=5)$, respectively. Tumour volume $\left(\mathrm{cm}^{3}\right)$ was calculated every week. After 7 weeks, each mouse was treated with $0.1 \mathrm{ml}$ cisplatin $(10 \mathrm{mg} \mathrm{kg}-1)$ via tail vein injection every 5 days and euthanized 10 days later. After complete cisplatin treatment, all mice were euthanized, and the tumours were excised and imaged under a light microscope $\left({ }^{*} \mathrm{P}\right.$ value $\left.<0.05\right)$ 
miR-363 into BALB/C nude mice. Thirty-five days after injection, when all mice developed palpable tumours, each mouse was treated with $0.1 \mathrm{ml}$ cisplatin $(10 \mathrm{mg} / \mathrm{kg})$ every 5 days and euthanized 10 days later. As shown in Fig. 2D, prior to cisplatin treatment, tumour growth was slightly inhibited in the miR-363 overexpressing group compared to the control group. Notably, upon 10-day cisplatin treatment, tumour growth was more dramatically inhibited in the miR-363 overexpressing group than in the control group (Fig. 2D). Taken together, findings suggest that miR-363 might sensitize EOC cells to cisplatin treatment.

\section{Snail is upregulated in cisplatin-resistant EOC cell lines and patients}

Previous reports revealed that induction of EMT may contribute to the decreased efficacy of cisplatin therapy. To examine EMT in response to cisplatin treatment, the expression levels of EMT-related markers (E-cadherin, fibronectin, N-cadherin, vimentin) and inducers (TCF- $\beta 1$,

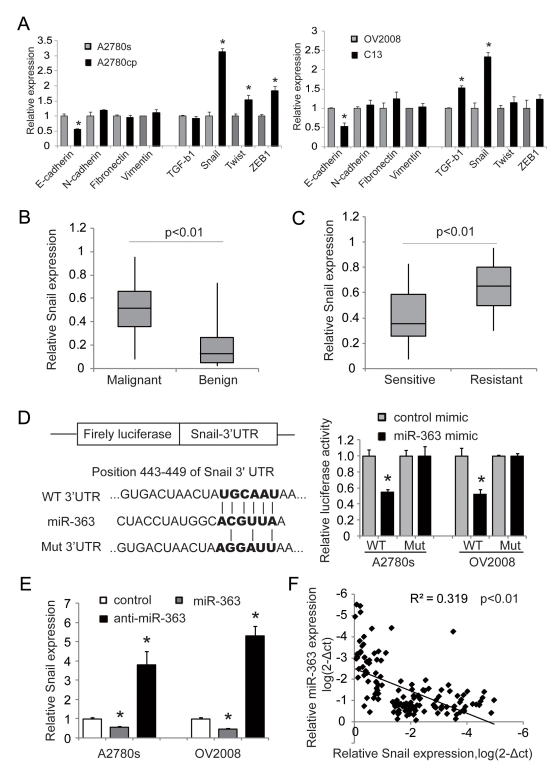

Fig. 3. Snail was upregulated in cisplatin-resistant EOC and its expression was inhibited in EOC cells by miR-363. (A) qRT-PCR analysis of the expression levels of EMT inducers in chemoresistant OVC cells A2780cp and C13 and their chemosensitive counterparts A2780s and OV2008 cells. (B) miR-363 expression levels in 107 malignant EOC tissues and 29 benign tissues. (C) miR-363 expression levels in primary cisplatin-sensitive $(n=72)$ and cisplatin-resistant $(\mathrm{n}=35)$ EOC tissues ( $\mathrm{P}$ value $<0.05)$. (D) Snail had a predicted miR-363 binding site in 3'UTR, and luciferase activity of the reporter construct containing the wild-type or mutant miR-363 binding site was measured after co-transfection with $50 \mathrm{nM}$ miRNAs. (E) qRT-PCR was performed to determine snail expression levels in miR-363 overexpression or knockdown cells (*P value $<0.05$ ). (F) The reverse relation of miR-363 and snail was observed in the correlation analysis of their expressions in 107 patients with malignant EOC. snail, slug, twist, ZEB1) were evaluated in chemoresistant A2780cp and C13 cells and their chemosensitive counterparts A2780s and OV2008 cells. qRT-PCR analysis revealed that E-cadherin was significantly downregulated in chemoresistant A2780cp and C13 cells compared with that in their chemosensitive counterparts A2780s and OV2008 cells (Fig. $3 \mathrm{~A})$, suggesting that EMT was promoted in response to cisplatin treatment. Among EMT inducers tested, snail was most upregulated in A2780cp and C13 cells compared with that in A2780s and OV2008 cells (Fig. 3A). In addition, snail expression levels in EOC and benign tissues were detected where Snail relative expression levels were found to be higher in malignant tissues compared with benign tissues (Fig. 3B) and were also higher in cisplatin-resistant patients compared with those in cisplatin-sensitive patients (Fig. 3C). Taken together, these data suggest that snail is upregulated in cisplatin-resistant EOC cell lines and patients.

\section{miR-363 directly inhibits snail expression in EOC cells}

Then in silico prediction for miR-363 target genes suggested that snail might be a target of miR-363 in EOC cells (Fig. 3D). To validate if miR-363 could directly target 3'UTR of snail, a fragment of wild-type or mutant 3'UTR of snail was cloned into psi-CHECK2 reporter vector, respectively. Luciferase reporter assays revealed that miR-363 significantly decreased the relative luciferase activity of 3'UTR of snail in A2780s and OV2008 cells but had no effect on the mutant 3'UTR of Snail. qRT-PCR analysis further revealed that miR-363 mimics

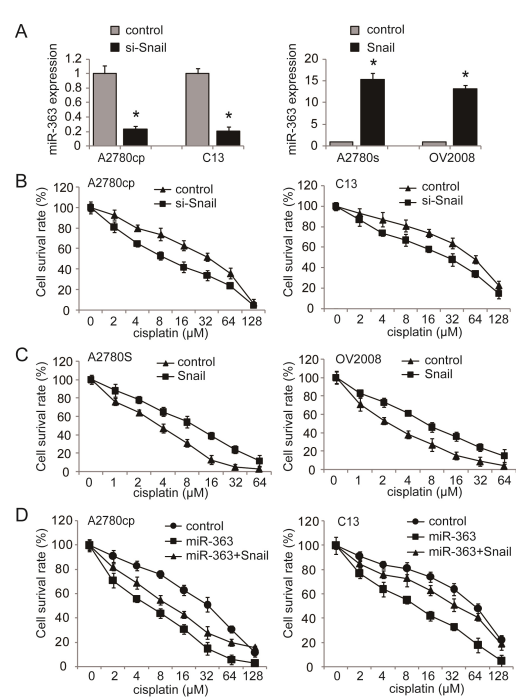

Fig. 4. Snail is involved in miR-363-suppressed cisplatinresistance of EOC cells. (A) qRT-PCR was performed to determine snail expression levels in snail knockdown cells (A2780cp and C13) and snail overexpression cells (A2780s and OV2008). (B, C, D) The impacts of snail and miR-363 on cisplatin sensitivity at different doses $(0,2,4,6,8$ and $10 \mathrm{ng} / \mathrm{ml})$ were determined by MTT assay ( ${ }^{*} P$ value $\left.<0.05\right)$. 
significantly reduced the snail expression, and knocking-down of miR-363 increased Snail expression in A2780s and OV2008 cells (Fig. 3E). The reverse relation of miR-363 and Snail was observed in correlation analysis of their expressions in 107 malignant EOC patients $\left(\mathrm{R}^{2}=0.319, \mathrm{P}<0.01\right)$ (Fig. 3F). Taken together, these data suggest that snail is a functional target of miR-363 in EOC cells.

\section{Snail is involved in miR-363-suppressed cisplatin-resistance of EOC cells}

To further determine whether miR-363 exerts its effect on regulating cisplatin sensitivity through the downregulation of snail in EOC cells, Snail in A2780cp and C13 cells were knocked down with snail-specific small interfering RNAs (si-Snail), and snail expression in A2780s and OV2008 cells was upregulated (Figs. 4A and S2). As expected, MTT assay revealed that snail knockdown significantly sensitized A2780cp and C13 cells to cisplatin (Fig. 4B), while snail upregulation resulted in decreased cisplatin sensitivities of A2780s and OV2008 cells (Fig. 4C). These observations were opposite the effects of miR-363. Then, snail expression was rescued in stable miR-363 overexpressing A2780cp or C13 cells by transfecting snail expression plasmids lacking 3'UTR. MTT assay revealed that Snail overexpression significantly attenuated miR-363-suppressed cisplatin resistance of A2780cp and $\mathrm{C} 13$ cells (Fig. 4D). Taken together, these results suggest that snail is a functional target of miR-363, involved in miR-363-suppressed cisplatinresistance of EOC cells.

\section{DISCUSSION}

Cisplatin is a first-line antitumour chemotherapeutic agent; however, due to cisplatinresistance of EOC, $25 \%$ of patients will develop resistance to this agent within 6 months after chemotherapy (22). To break the therapeutic barrier of cisplatin resistance in EOC, it is urgent to clarify the mechanisms underlying cisplatin resistance and identify new biomarker and therapeutic targets.

Recently, multiple studies have indicated that miRNA dysregulation played an important role in the development of cisplatin resistance in EOC. The human let-7 family is well known to associate with tumorigenesis of different types of cancers, such as lung cancer, breast cancer, prostate cancer and OVC (23-26). It was reported that let-7e expression was significantly reduced in cisplatin-resistant EOC cell line and let-7e overexpression could resensitize cisplatin-resistant EOC cells to cisplatin (27). In this study, we have shown that miR-363 levels were significantly downregulated in malignant EOC tissues compared with benign tissues, and miR-363 downregulation was significantly correlated with high FIGO stage, metastasis and chemoresistance. Moreover, miR-363 overexpression could resensitize cisplatin-resistant EOC cells, and miR-363 knockdowncould decrease the cisplatin sensitivities of normal EOC cells both in vitro and in vivo.
The upregulation of EMT inducers was supposed to be closely associated with the development of multiple chemotherapeutic drug resistance in different cancers $(28,29)$. Haslehurst et al. found that the EMT inducer snail was upregulated in the chemoresistant EOC cells (30). By evaluating expressions of EMT inducers in both chemoresistant EOC cells and their chemosensitive counterparts, it was found that snail was highly upregulated in chemoresistant EOC cells and cisplatin-resistant patients. In addition, as predicted, snail might be a target of miR-363 with in silico study. The subsequent luciferase reporter assays proved the prediction, and an inverse correlation between miR-363 and Snail expression was observed in both EOC cells and patients. It was reported that knockdown of snail leads to cisplatin sensitization in lung adenocarcinoma and head and neck squamouscell carcinoma $(28,31)$. Results revealed that snail knockdown significantly sensitized EOC cells to cisplatin and snail upregulation resulted in decreased cisplatin sensitivities. Furthermore, rescued snail expression in miR-363 overexpressing EOC cells could significantly attenuate miR-363suppressed cisplatin resistance of these cells. It was seem that snail couldn't fully restoring cell survival under cisplatin treatment condition. As we all known, each miRNA may regulates multiple (even hundreds) of target genes, there may be other target of miR-363 that affects cisplatin resistance in EOC cell lines. Just as reported, FBW7, MCl-1, ABCC1 were target genes for miR-363 in regulating chemoresistance (14, $16,21)$. In OC, miR-363 play a tumor suppressor role by targeting NOB1(20), these are possible targets of miR-363 which affects cisplatin resistance in EOC, but we need to verify it.

In conclusion, this study provides evidence that miR-363 could sensitize EOC cells to cisplatin treatment in vitro and in vivo. More importantly, the data demonstrate for the first time that snail is a target of miR-363, and MiR-363 inhibits cisplatin resistance of EOC by regulating snail-induced EMT. Taken together, findings indicate that miR-363 may be a biomarker for predicting responsiveness to cisplatin-based chemotherapy and a potential therapeutic target in EOC.

\section{MATERIALS AND METHODS}

\section{Patients and samples}

One hundred seven patients with OVC were enrolled at Xiangya Hospital (Changsha, Hunan, China) from 2013 to 2016. All patients were given written informed consent, and this study was authorized by the Ethics Committee of Xiangya Hospital, South University. Treatment response was assessed with clinical and radiologic examination evaluated by the same investigator according to RECIST (Response Evaluation Criteria in Solid Tumours, version 1.0). The responders were defined as having either complete or partial response. The non-responders included patients with stable or progressive disease. 


\section{Cell culture}

Csiplatin sensitive (OV2008, A2780s) and resistant (C13, A2780cp) were generously provided by Drs. Rakesh Goel and Barbara Vanderhyden (Ottawa Hospital Cancer Center, Ottawa, ON, Canada). Cells were cultured at $37^{\circ} \mathrm{C}, 5 \% \mathrm{CO}_{2}$ in either RPMI 1640 (OV2008 and C13) or DMEM-F12 (A2780s and A2780-cp) containing 10\% FBS (Invitrogen, USA). Cells (1 $\times 10^{6}$ ) were plated in log growth phase onto 60 -mm dishes for $24 \mathrm{~h}$ in the above culture medium before the initiation of treatment.

\section{qRT-PCR}

Total RNAs were extracted using the TRIzol method (Invitrogen, USA) and reversely transcribed into cDNA using the PrimeScript RT reagent Kit (TaKaRa Bio, Japan) according to the manufacturer's instructions. qRT-PCR was performed on ABI 7500 Sequence Detection System (Life Technologies, USA) using SYBR Green real-time PCR master mix (Toyobo Co., Japan). The specific primers for miRNA-363 and small nuclear U6, which was used as an internal control, were purchased from Guangzhou RiboBio (Guangzhou RiboBio Co., Ltd., Guangzhou, China). Relative expression levels were calculated using the $2^{-\Delta \Delta C t}$ method. Primers for qRT-PCR were synthesized by Invitrogen (Shanghai, China); the sequences are listed in Supplementary Table 2.

\section{Cell proliferation assay}

The cell proliferation was assessed using 3-(4,5-dimethylthiazol-2-yl)-2,5-diphenyltetrazoliumbromide (MTT) solution (Sangon Biotech, China). Forty-eight h after transfection, EOC cells were seeded into 96-well plates at an initial density of 5 $\times 10^{3}$ cells/well. After $24 \mathrm{~h}$ of culture, these cells were exposed to various concentrations of cisplatin for $24 \mathrm{~h}$, respectively. Then the cells were treated with $10 \mu \mathrm{MTT}$ by adding it into each well. The cells were incubated at $37^{\circ} \mathrm{C}$ with $5 \% \mathrm{CO}_{2}$ for another $4 \mathrm{~h}$; then the medium was removed carefully, and 150 $\mu$ l dimethylsulfoxide (DMSO) solution (MP Biomedicals, USA) was added for $10 \mathrm{~min}$ to lyse the cells. Subsequently, the absorbance was measured at $570 \mathrm{~nm}$ using a microplate reader Multiskan MK (Thermo Scientific, USA). The survival rate was calculated using the equation: (mean absorbance of drug well $/$ mean absorbance of control wells) $\times 100 \%$.

\section{Lentiviral infection}

Knocking-down miR-363 by the lentiviral GV-428 vector, encoding the anti-sense of miR-363 (anti-miR-363, based on the described sequence [23]) was designed, synthesized and sequence-verified by the GeneChem Company (Shanghai, China). Lentivirus-expressing miR-363 (Lv-miR-363) and negative control (Lv-NC) were also purchased from the GeneChem Company. To get stably infected cells, the cells were cultured in about $80 \%$ of the plates and then added by a concentration of $5.0 \times 10^{4} \mathrm{TU} /$ well lentivirus. RTq-PCR was performed to determinate miR-363 expression levels after being infected for 5 days.

\section{Animal treatment}

All animal experiments were carried out in accordance with a protocol approved by the Institutional Ethical Committee (Institutional Animal Care and Use Committee of Xiangya Hospital). Five-week-old BALB/c nu/nu mice were purchased from Shanghai Laboratory Animal Center (SLAC, Shanghai, China) and were handled under specific pathogen-free conditions. A2780cp cells overexpressing miR-363 $\left(5 \times 10^{6}\right.$ cells in $0.1 \mathrm{ml}$ of phosphate-buffered saline per mouse) were injected into the proximal tibia of each mouse $(n=5$ animals per group). Every week post inoculation, the individual tumour was measured with calipers according to the formula: $1 / 2 \times$ length $\times$ width $^{2}$. Seven weeks after inoculation, when all mice developed palpable tumours, each mouse was treated with 0.1 $\mathrm{ml}$ cisplatin $\left(10 \mathrm{mg} \mathrm{kg}^{-1}\right)$ via tail vein injection once a week for 2 weeks. After complete cisplatin treatment, all of the mice were euthanized, and the tumours were excised and imaged under a light microscope.

\section{Dual-luciferase reporter assay}

The fragments from 3'UTR of snail containing the predicted miR-363 binding site were synthesized and cloned into the luciferase construct psi-CHECK2. The resulted vector snail3'UTR-psi-CHECK2 was called the reporter vector WT-3'UTR. The corresponding mutant was called Mut-3'UTR. The miR-363 mimic or control mimic was co-transfected with the reporter vectors using transfection reagent (Invitrogen, USA). $48 \mathrm{~h}$ after transfection, Firefly and Renilla luciferase activities in cell lysates were measured using the Dual-Luciferase Reporter Assay Kit (E1910; Promega, USA).

\section{Statistical analysis}

The experiments were repeated at least 3 times, and the data are shown as the mean \pm s.d. Data analyses were performed using Student's t-test for simple comparison of the 2 groups. The difference in results was considered statistically significant when the $\mathrm{P}$ value was $<0.05$.

\section{ACKNOWLEDGEMENTS}

This work was supported by Key research and development project of Hunan provincial science and Technology Department (2017sk2071) and the National Natural Science Fundation of China (81570776).

\section{CONFLICTS OF INTEREST}

The authors have no conflicting interests.

\section{REFERENCES}

1. Chiu WT, Huang YF, Tsai HY et al (2015) FOXM1 confers 
to epithelial-mesenchymal transition, stemness and chemoresistance in epithelial ovarian carcinoma cells. Oncotarget 6, 2349-2365

2. Kuchenbaecker KB, Ramus SJ, Tyrer J et al (2015) Identification of six new susceptibility loci for invasive epithelial ovarian cancer. Nat Genet 47, 164-171

3. Raghavan R, Hyter S, Pathak HB et al (2016) Drug discovery using clinical outcome-based Connectivity Mapping: application to ovarian cancer. BMC Genomics 17,811

4. Siddik ZH (2003) Cisplatin: mode of cytotoxic action and molecular basis of resistance. Oncogene 22, 7265-7279

5. Zamble DB and Lippard SJ (1995) Cisplatin and DNA repair in cancer chemotherapy. Trends Biochem Sci 20, 435-439

6. Siegel R, Naishadham D and Jemal A (2012) Cancer statistics for Hispanics/Latinos, 2012. CA Cancer J Clin 62, 283-298

7. Polyak K and Weinberg RA (2009) Transitions between epithelial and mesenchymal states: acquisition of malignant and stem cell traits. Nat Rev Cancer 9, 265-273

8. Marchini S, Fruscio R, Clivio L et al (2013) Resistance to platinum-based chemotherapy is associated with epithelial to mesenchymal transition in epithelial ovarian cancer. Eur J Cancer 49, 520-530

9. Calin GA and Croce CM (2006) MicroRNA signatures in human cancers. Nat Rev Cancer 6, 857-866

10. Chang TC, Yu D, Lee YS et al (2008) Widespread microRNA repression by Myc contributes to tumorigenesis. Nat Genet 40, 43-50

11. Johnson CD, Esquela-Kerscher A, Stefani G et al (2007) The let-7 microRNA represses cell proliferation pathways in human cells. Cancer Res 67, 7713-7722

12. Tsai WC, Hsu SD, Hsu CS et al (2012) MicroRNA-122 plays a critical role in liver homeostasis and hepatocarcinogenesis. J Clin Invest 122, 2884-2897

13. Liu J, Li Q, Li R, Ren P and Dong S (2017) MicroRNA363-3p inhibits papillary thyroid carcinoma progression by targeting PIK3CA. Am J Cancer Res 7, 148-158

14. Zhang PF, Sheng LL, Wang G et al (2016) miR-363 promotes proliferation and chemo-resistance of human gastric cancer via targeting of FBW7 ubiquitin ligase expression. Oncotarget 7, 35284-35292

15. Hu F, Min J, Cao X et al (2016) MiR-363-3p inhibits the epithelial-to-mesenchymal transition and suppresses metastasis in colorectal cancer by targeting Sox4. Biochem Biophys Res Commun 474, 35-42

16. Zhang R, Li Y, Dong X, Peng L and Nie X (2014) MiR-363 sensitizes cisplatin-induced apoptosis targeting in Mcl-1 in breast cancer. Med Oncol 31, 347

17. Li Y, Chen D, Li Y et al (2016) Oncogenic cAMP responsive element binding protein 1 is overexpressed upon loss of tumor suppressive miR-10b-5p and
miR-363-3p in renal cancer. Oncol Rep 35, 1967-1978

18. Chen Y, Lu X, Wu B, Su Y, Li J and Wang H (2015) MicroRNA 363 mediated positive regulation of c-myc translation affect prostate cancer development and progress. Neoplasma 62, 191-198

19. Conti A, Romeo SG, Cama A et al (2016) MiRNA expression profiling in human gliomas: upregulated miR-363 increases cell survival and proliferation. Tumour Biol 37, 14035-14048

20. Lin $Y, X u T$, Zhou $S$ and Cui $M$ (2017) MicroRNA-363 inhibits ovarian cancer progression by inhibiting NOB1. Oncotarget 8, 101649-101658

21. Huang $H$, Chen J, Ding CM, Jin X, Jia ZM and Peng J (2018) LncRNA NR2F1-AS1 regulates hepatocellular carcinoma oxaliplatin resistance by targeting $A B C C 1$ via miR-363. J Cell Mol Med 22, 3238-3245

22. Zhang $P$, Zhang $P$, Shi $B$ et al (2014) Galectin-1 overexpression promotes progression and chemoresistance to cisplatin in epithelial ovarian cancer. Cell Death Dis 5, e991

23. Boyerinas B, Park SM, Murmann AE et al (2012) Let-7 modulates acquired resistance of ovarian cancer to Taxanes via IMP-1-mediated stabilization of multidrug resistance 1. Int J Cancer 130, 1787-1797

24. Chin LJ, Ratner E, Leng $S$ et al (2008) A SNP in a let-7 microRNA complementary site in the KRAS 3' untranslated region increases non-small cell lung cancer risk. Cancer Res 68, 8535-8540

25. Liu C, Kelnar K, Vlassov AV, Brown D, Wang J and Tang DG (2012) Distinct microRNA expression profiles in prostate cancer stem/progenitor cells and tumor-suppressive functions of let-7. Cancer Res 72, 3393-3404

26. $Y u$ F, Yao $H$, Zhu $P$ et al (2007) let-7 regulates self renewal and tumorigenicity of breast cancer cells. Cell $131,1109-1123$

27. Cai J, Yang C, Yang Q et al (2013) Deregulation of let-7e in epithelial ovarian cancer promotes the development of resistance to cisplatin. Oncogenesis 2, e75

28. Hsu DS, Lan HY, Huang CH et al (2010) Regulation of excision repair cross-complementation group 1 by Snail contributes to cisplatin resistance in head and neck cancer. Clin Cancer Res 16, 4561-4571

29. Kaufhold S and Bonavida B (2014) Central role of Snail1 in the regulation of EMT and resistance in cancer: a target for therapeutic intervention. J Exp Clin Cancer Res 33, 62

30. Haslehurst AM, Koti $M$, Dharsee $M$ et al (2012) EMT transcription factors snail and slug directly contribute to cisplatin resistance in ovarian cancer. BMC Cancer 12, 91

31. Zhuo W, Wang $Y$, Zhuo $X$, Zhang $Y$, Ao $X$ and Chen $Z$ (2008) Knockdown of Snail, a novel zinc finger transcription factor, via RNA interference increases A549 cell sensitivity to cisplatin via JNK/mitochondrial pathway. Lung Cancer 62, 8-14 